\title{
Cognitive dysfunction in diabetes mellitus
}

\section{Introduction}

Some of the well-known long term complications of diabetes are polyneuropathy, cranial neuropathy, cerebrovascular accidents.

Diabetes is associated with smaller brain volume in grey but not white matter. Numerous domains of cognitive function are impaired in both type-1 and type-2 DM. Hyperglycemia, hypoglycemia, vascular changes and insulin resistance play significant role in their causation.

\section{Types of impaired cognitive function in DM (type-I and type-2)}

During cognitive function test following changes are seen. Slowing of information processing, worsening of psychomotor efficiency, motor speed, motor strength, vocabulary, general intelligence, visual construction, attention, somato sensory, memory and executive function are impaired.

\section{Pathophysiology of cognitive dysfunction in diabetes}

Hyperglycemia, hypoglycemia, vascular disease, insulin resistance and amyloid deposition are few which are implicated in causation of cognitive dementia (Figure 1).

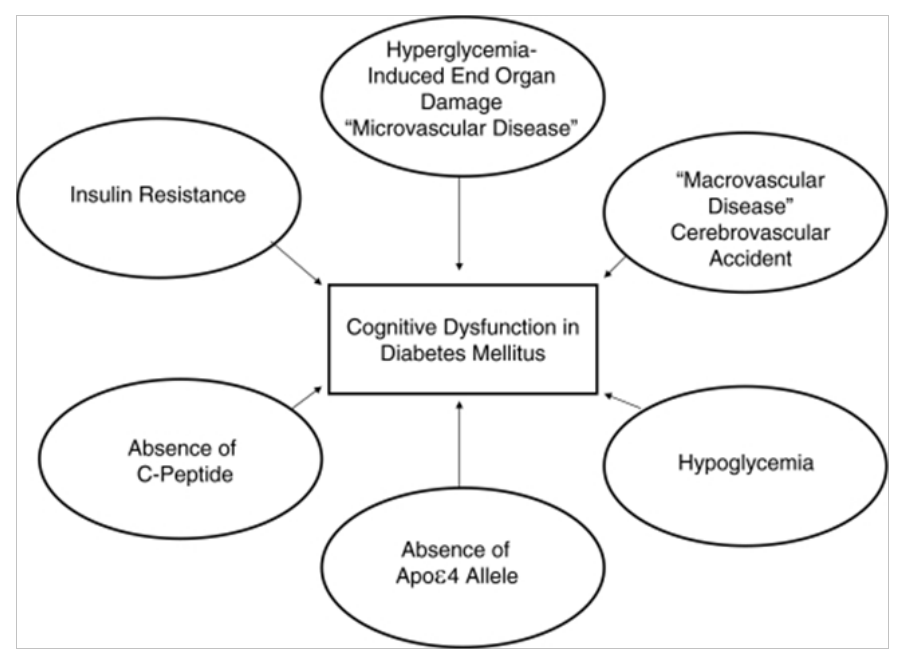

Figure I Hyperglycemia, hypoglycemia, vascular disease, insulin resistance and amyloid deposition are few which are implicated in causation of cognitive dementia.

\section{Brain atrophy}

There is decrease in the total volume of grey, white and hippocampal gyrus. Grey matter loss was seen over medial temporal, anterior cigulate, medial frontal lobe. White matter loss was in temporal and frontal region. Cortical atrophy in T2DM resembles pattern seen in preclinical Alzheimer disease. Neurodegenerative rather cerebrovascular lesion may play a role in T2DM related cognitive impairment. ${ }^{2}$

\section{Hypoglycemia and cognitive disorders}

Better glycemic control improves cognitive function. ${ }^{3}$ Acute
Volume 6 Issue I - 2019

\author{
Raman PG \\ MGM Medical College, India
}

Correspondence: Pondy GR Raman, MGM Medical College, India, Email drpgraman@yahoo.com

Received: January 24, 2019 | Published: February 07, 2019

hyperglycemia worsens cognitive function. In one study, distal symmetrical polyneuropathy displayed worst cognitive function except memory. ${ }^{4}$ Onset of cognitive dysfunction is found to occur within two years from the onset of type 1 diabetes. Type-2 DM patients also have cognitive dysfunction. The impacts of these deficits in daily lives of patients produce considerable problems. Clinically, these patients have imbalance and slow walking and in type 2 diabetes. Alzheimer's disease is associated. There is greater risk of vascular dementia, ${ }^{5,6} \mathrm{HbA1C}$ over 7 showed 4 fold increase in cognitive impairment. There is association between $\mathrm{HbA1C}$, duration of DM and peripheral neuropathy to impair cognitive function.

\section{Role of vascular disease}

DM has 2-6 fold increase in thrombotic stroke. Autopsy studies in long standing type-1 diabetes mellitus showed diffused brain degeneration, psuedocalcinosis and demyelination affecting spinal cord and cranial nerve. ${ }^{\top}$

Due to decrease in cerebral blood flow, inadequate cerebral vasodilatation occurs in $\mathrm{DM}$ along with thromboxane $\mathrm{A} 2$ receptor stimulation. Blood glucose over $8.6 \mathrm{~m} . \mathrm{mol} / \mathrm{lit}$ during CVA correlate with poor clinical recovery. Due to cellular acidosis provided by substrate lactate as a result of hyperglycemia there is brain injury. Hyperglycemia leads to accumulation of glutamate is an excitary amino acid neuron transmitter which can cause neuronal damage. ${ }^{8,9}$

\section{The role of hypoglycemia}

In younger type-1 DM less than 5 years who had seizures during frequent hypoglycemia showed impaired cognitive function. It seems younger patients' brain was more susceptible to injury and develop cognitive dysfunction. ${ }^{10}$ Brain death and damage can occur if hypoglycemia lasts for prolonged period. ${ }^{11}$ In animal models after $30-60 \mathrm{~min}$ of blood glucose levels between 0.12 and $1.36 \mathrm{mmol} /$ liter, neuronal necrosis occurs. Flat electroencephalograph (EEG) is seen in this group of animals. Laminar necrosis and gliosis were found in cortex, basal ganglia, and hippocampus due to hypoglycemia in human patients who died. ${ }^{12}$ Multifocal or diffuse necrosis of the cerebral cortex and chromatolysis of ganglion cells are seen in humans due to hypoglycemia. On the other hand in animals, hypoglycemia-induced damage seems to be selective to neurons with sparing of astrocytes and oligodendrocytes. There may be a relationship to hypoglycemia during early nocturnal sleep, a time in which consolidation of memories and cognitive dysfunction occurs. 


\section{The role of insulin resistance and amyloid in diabetes and alzheimer's disease}

Alzheimer's disease is diagnosed in the presence of progressive decrease in memory and it has been found approximately 1.2 to 1.7 fold higher in type 2 diabetes.

Cerebro spinal fluid level is decreased in Alzheimer's suggesting impaired insulin transport across blood brain barrier. ${ }^{13}$ Alzheimer's patients have decreased cerebral glucose uptake and reduced number of glucose transporters compared with controls on autopsy studies. Type 2 diabetes mellitus and insulin resistance may contribute to cognitive dysfunction through other indirect mechanisms. Inflammatory markers and increased inflammation contribute to cognitive dysfunction in patients with type 2diabetes and development of macro vascular disease. ${ }^{14}$

Hypothalamic-pituitary adrenal axis is disrupted contributes to cognitive dysfunction in type 2 diabetes. Hypercortisolemia causes cognitive dysfunction both in animals and humans with type 2 diabetes.

By promoting the formation of senile plaques with insulin resistance, cognitive dysfunction can occur. The pathological signs of Alzheimer's disease are extracellular senile plaques composed of $\beta$-amyloid and intracellular neurofibrillary tangles. Amyloid precursor protein (APP), which is produced in neurons, by the enzymes $\beta$-and $\gamma$-secretase, gives rise to $\beta$-Amyloid from its cleavage. The insulindegrading enzyme eventually degrades $\beta$-Amyloid.

It is still under investigation that Alzheimer's disease can be treated with peroxisome proliferator-activated receptor- $\gamma$ (PPAR- $\gamma$ ) in the absence of diabetes. Rosiglitazone has a beneficial effect on memory in patients with Alzheimer's disease.

\section{Modalities for assessment of cognitive dysfunction in patients with diabetes I 5, 16}

Measure the electrical activity such as evoked response potentials in the brain after the administration of different stimuli. Abnormal evoked response potentials can reveal subclinical sensory nerve conduction deficits that may not otherwise be apparent. In diabetes, brainstem auditory-evoked potentials demonstrated acoustic pathway impairment.

EEG has been used in patients with type 1 and type 2 diabetes and can also detect spontaneous cerebral electrical activity. Type 2 diabetes patients show slow EEG frequency over the central cortex area and reduction of alpha activity over the parietal area along with reduced visual retention on neurocognitive testing. Type 1 diabetes patients were found to have abnormal EEG when compared with controls. Those patients with a history of severe hypoglycemia showed most abnormalities.

To examine cerebral structure in patients with type 1 and type 2 diabetes Magnetic resonance imaging (MRI) has been used in many studies. The brains of such subjects have leukoariosis, which are hyper intense white matter lesions. In a study, abnormal MRI scans were found in $69 \%$ of middle-aged adults with long-standing type 1diabetes, compared with $12 \%$ of healthy, aged-matched volunteers, with an increased number of larger, high-signal lesions in the cerebrum, cerebellum, and brain stem being the primary abnormality identified.

Type-2 DM and IFG are increased in Alzheimer. There is increased frequency of islet amyloid in Alzheimer. Brain amyloid was not increased in type-2 DM but with long duration of diabetes, there was increased density of the diffuse neuritic plaques. These deposits are cytotoxic may disrupt membrane and damage islet cells in diabetes and brain cells in Alzheimer. ${ }^{17,18}$

\section{Conclusion}

Cognitive dysfunction should be listed as one of the many complications of diabetes, along with retinopathy, neuropathy, nephropathy, and cardiovascular disease. The pathogenesis of cognitive dysfunction is only partially understood. Although many studies suggest that changes in cerebral structure and function in diabetes are related to hyperglycemia-induced end organ damage. Macro vascular disease, hypoglycemia, insulin resistance, and amyloid lesions may play a role in some patients. Greater understanding of the natural history of diabetes complications and the mechanisms responsible for their development will continue to advance as biochemical and imaging modalities continue to evolve.

\section{Acknowledgment}

None.

\section{Conflicts of interest}

The author declares no conflict of interest.

\section{References}

1. Kodi CT. Cognitive Dysfunction and Diabetes Mellitus. Endocr Review. 2008;(4):494-511.

2. Mecrimmon RJ, Ryan CM, Frier BM. Diabetes and Cognitive Dysfunction. Lancet. 2012;379:2291-2299.

3. Jacobson AM, Musen G, Ryan CM, et al. Long-term effect of DM and its treatment on cognitive function. $N$ Engl J Med. 2007;356:1842-1852.

4. Ryan CM, William TM, Finegold DN, e al. Cognitive dysfunction in adults with type-1 DM of long duration, effect of recurrent hypoglycemia and other chronic complications. Diabetologia. 1993;36:329-334.

5. Ott A, Stolk RP, Hofman A, et al. Association of DM and Dementia: The Rotterdam Study. Diabetologia. 1996;39:1392-1397.

6. Yaffe K, Blackwell T, Whitmer RA, et al. Glycosylated Hb Level and Development of mild cognitive impairment or dementia in older women. Journal Nutri Health Aging. 2006;10:293-295.

7. Reske NE, Lundbeck K. Pathological changes in central and peripheral nervous system in young, long-term diabetics II Spinal cord and peripheral nerves. Diabetologia. 1968;4:34-43.

8. McCall AL. The Impact of DM on CNS. Diabetes. 1992;4:557-570.

9. Coyle JP, Puttfarken P. Oxidative Stress, Glutamate and Neurodegenerative Disorders. Science. 1993;262:689-695.

10. Ryan C, Vega A, Drash A. Cognitive deficits in adolescents who developed DM early in life. Pediatriacts. 1985;75:921-927.

11. Patrick AW, Campbell IW. Fatal hypoglycaemia in insulin-treated diabetes mellitus: clinical features and neuropathological changes. Diabet Med. 1990;7:349-354.

12. Auer RN. Hypoglycemic brain damage. Metab Brain Dis. 2004;19:169175.

13. Bucht G, Adolfsson R, Lithner F, et al. Changes in blood glucose and insulin secretion in patients with senile dementia of Alzheimer type. Acta Med Scand. 1983;213:387-392. 
14. Craft S, Peskind E, Schwartz MW, et al. Cerebrospinal fluid and plasma insulin levels in Alzheimer's disease: relationship to severity of dementia and apolipoprotein E genotype. Neurology. 1998;50:164-168.

15. Mirzaei S, Gelpi E, Booij J, et al. New approaches in nuclear medicine for early diagnosis of Alzheimer's disease. Curr Alzheimer Res. 2004;1:219-229

16. Mosconi L, De Santi S, Li Y, et al. Visual rating of medial temporal lobe metabolism in mild cognitive impairment and Alzheimer's disease using FDG-PET. Eur J Nucl Med Mol Imaging. 2006;33:210-221.
17. Tojo C, Takao T, Nishioka T, et al. Hypothalamic-pituitary-adrenal axis in WBN/Kob rats with non-insulin dependent diabetes mellitus. Endocr J. 1996;43:233-239.

18. Masters CL, Multhaup G, Simms G, et al. Neuronal origin of a cerebral amyloid: neurofibrillary tangles of Alzheimer's disease contain the same protein as the amyloid of plaque cores and blood vessels. EMBO J. $1985 ; 4: 2757-2763$ 